\title{
Gynecologic Ultrasound as a Pharmacosurveillance Tool
}

\section{Dale Quest}

Associate Professor of Pharmacology, Department of Medical Education, Paul L Foster School of Medicine, Texas Tech University Health Sciences Center, 5001 El Paso Drive, El Paso, Texas 79905, USA

Correspondence: Dale Quest, Associate Professor of Pharmacology, Department of Medical Education, Paul L Foster School of Medicine, Texas Tech University Health Sciences Center, 5001 El Paso Drive, El Paso, Texas 79905, USA Phone: (915) 783-1700 Ext. 237, e-mail: dale.quest@ttuhsc.edu

\begin{abstract}
A traditional medical school curriculum does not formally prepare physicians to participate in evaluation of safety and effectiveness after medications enter the market. This article provides examples where ultrasound has unrealized potential as a noninvasive tool for longitudinal assessment of drug safety and effectiveness in the real-world. The examples are intended to sensitize medical schools and medical students to opportunities for physicians to advance therapeutic evaluation of drugs as they are used for approved and unapproved indications in actual practice.
\end{abstract}

Keywords: Gynecology, ultrasound, medical education, pharmacosurveillance, safety, effectiveness.

Efficacy describes how a drug performs under optimum clinical trial conditions, and is taken to estimate effectiveness in actual practice. Similar to most industrialized countries, the system for drug evaluation in the United States consists of premarketing efficacy trials of relatively short duration, that tend to include about 1,500 to 4,000 highly selected, homogeneously uncomplicated cooperative patients closely monitored by highly specialized physicians. ${ }^{1}$ Efficacy trials address a few highly specific, most often surrogate outcomes chosen by the manufacturer to satisfy regulatory requirements for authorization to market the drug. ${ }^{2}$ Not all actual or potential risks will be known at the time a drug enters clinical use. There may be subsets of patients for whom the risk is greater than it appears to be for the target population as a whole. ${ }^{3}$ Preclinical trials tend to over-estimate clinical benefit and under-estimate potential harm to the broader population of patients receiving the drug in actual clinical practice. Monitoring programs for the period after a medication enters the market have traditionally focused on pharmacovigilance, which is directed at identifying unanticipated or severe harm. ${ }^{1}$ Pharmacosurveilance refers to monitoring populations for benefit and harm related to medications used in the context of real world clinical practice. ${ }^{4}$ For several decades, the mechanism to evaluate the real world safety and effectiveness of medications after they enter the market has relied substantially on ad hoc case reporting. Reporting rates are estimated to be less than ten percent. ${ }^{5}$ Case reports are a very low level of evidenceoften poorly described in terms of patient characteristics and disease status. No denominator of drug use is available to judge the frequency of the cases, which should be the numerator, so estimates of the rates of harm are unobtainable. The inadequacies of a voluntary adverse drug reaction reporting system are universally recognized, and are underscored by widely publicized withdrawals of popular drugs because of fatal or near-fatal effects that were not detected during clinical trials prior to marketing. ${ }^{1}$

The Food and Drug Administration Amendments Act (FDAAA) of 2007 intends to strengthen the FDA's role and improve the system for assuring the safe and appropriate use of drugs after they are marketed. ${ }^{6}$ Still, the main mechanism for detecting adverse drug effects and to determine whether the efficacy demonstrated in clinical trials will similarly affect health outcomes in actual practice hinges on spontaneous voluntary reporting by physicians.

Imagine postgraduate surveillance of medical schools, comparing graduates of a school that actively prepares students to become engaged in pharmacosurveillance to the general run of schools that are passive. Might formal medical educational efforts make a substantial difference in the rate and extent that drug safety and effectiveness is evaluated in clinical practice? Can medical education drive the capacity 
and motivation of future physicians to play a more active role in assessing whether the treatments they prescribe are the safest and most effective therapeutic choices for their patients?

Multisite, international collaborations among clinicians and clinical groups are well-established for research into specific conditions such as cancer and diabetes. One of the best examples is the Children's Oncology Group (COG), an organization that has treated and monitored children with cancer for more than 40 years. Over 200 medical institutions in the US and many in Canada participate in the group. Each institution has a multidisciplinary team of clinicians for diagnosis, treatment, and surveillance of all principal cancers of infants, children, and adolescents-over 40,000 patients. ${ }^{2}$ Could such an initiative be undertaken in gynecology? Considering the advancements that have been made in the diagnostic and research use of ultrasound, gynecology has a remarkable tool for pharmacosurveillence: noninvasive, widely accessible, known to provide usefully detailed resolution of structure and function in up to four dimensions, and versatile in the scope of its application without directly influencing the condition it is being used to assess.

This article is not a comprehensive overview. The aim is to provide insight by way of examples of how future physicians could apply ultrasound to strengthen the evaluation of drug therapy for gynecological disorders.

One of the most useful examples is the application of color Doppler ultrasound to assess responses to medical treatment for uterine leiomyomata. Definitive treatment of leiomyomata is surgical, but drug therapies have a role as adjuncts and possibly as a monitored alternative. ${ }^{6}$ The rate and magnitude of response to gonadotropin-releasing hormone super agonists can purportedly range from no detectable change up to a $90 \%$ reduction in size over a period of 8-12 weeks. ${ }^{7}$ Noting that larger submucous and subserous leiomyomata are particularly responsive, variation in response is hypothesized to depend on differences in size, location, vascularity, impedence to blood flow ${ }^{8}$ (Figs 1 to 3$)$. These parameters could provide an index to stratify candidates for medical management, for vaginal, laparoscopic or abdominal myomectomy/hysterectomy. It could also evolve into a validated standard for comparing therapeutic efficiencies of different classes of drugs, including in particular, GnRH super agonists in relation to the newer GnRH antagonists, mifepristone, danazol, longacting progestins, aromatase inhibitors or somatostatin

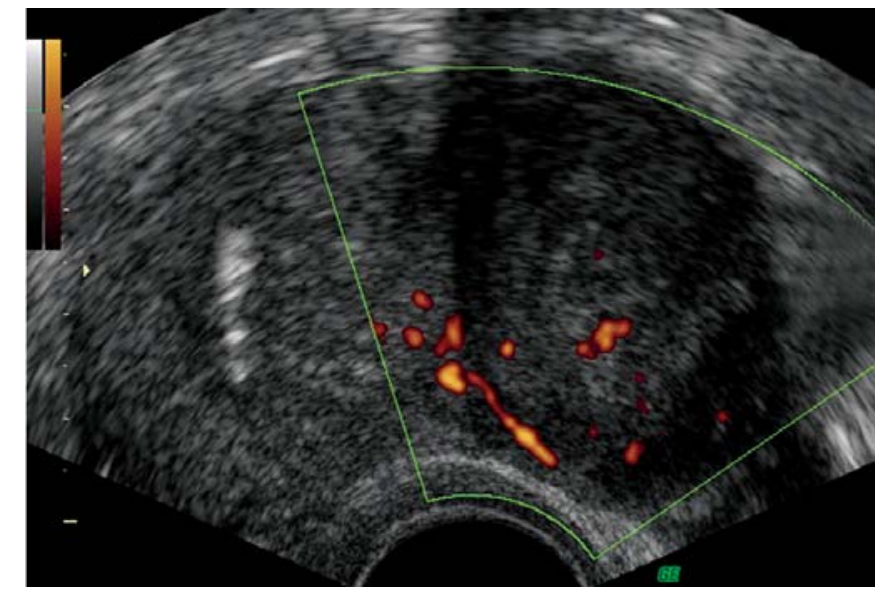

Fig. 1: 2-D color Doppler of the uterine artery branches supplying a subserosal fibroid (right)

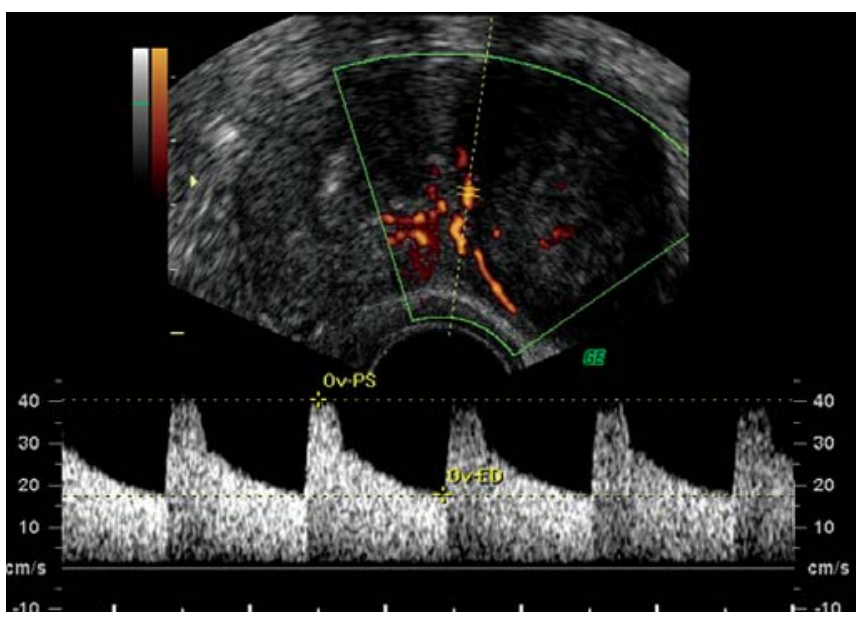

Fig. 2: Moderate vascular impedance blood flow signals are isolated from the fibroid vessels using pulsed Doppler waveform analysis

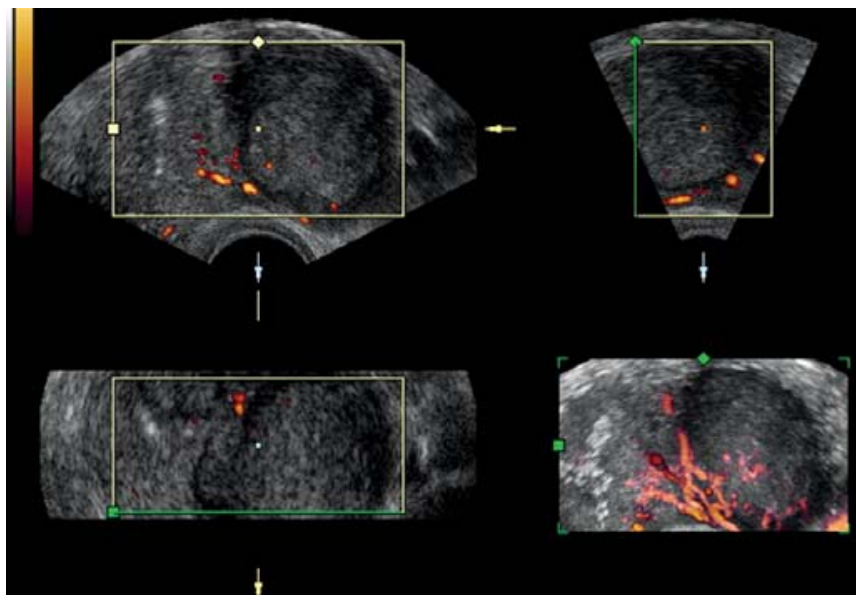

Fig. 3: 3-D power Doppler ultrasound of the uterine fibroid vessels

analogues as monotherapies and combinations to achieve the most desirable outcomes.

Color and spectral Doppler and transvaginal 3-D ultrasound provide means to stratify patients for surgical $v s$ 


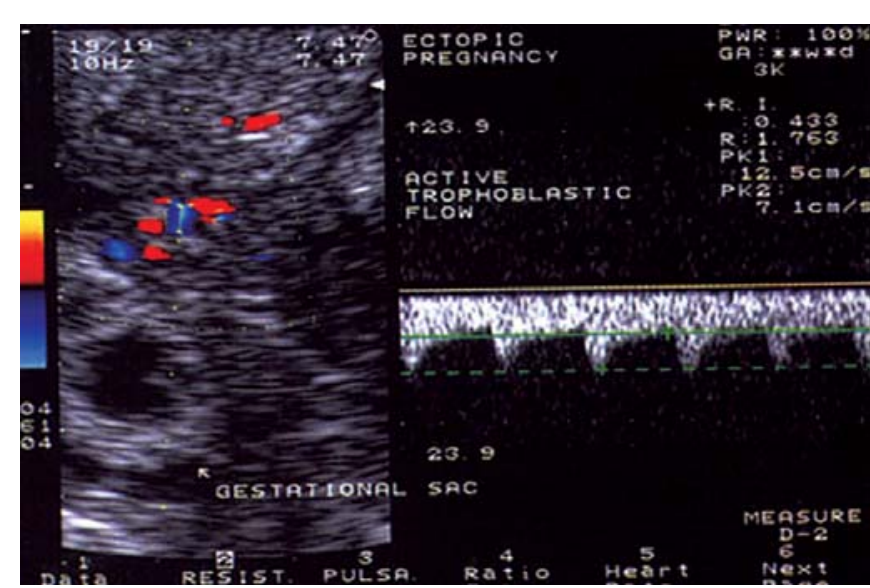

Fig. 4: Tubal ectopic pregnancy in a patient with positive pregnancy test and empty uterus. Low vascular impedance blood flow signals (RI of 0.43 ) are typical for active trophoblast

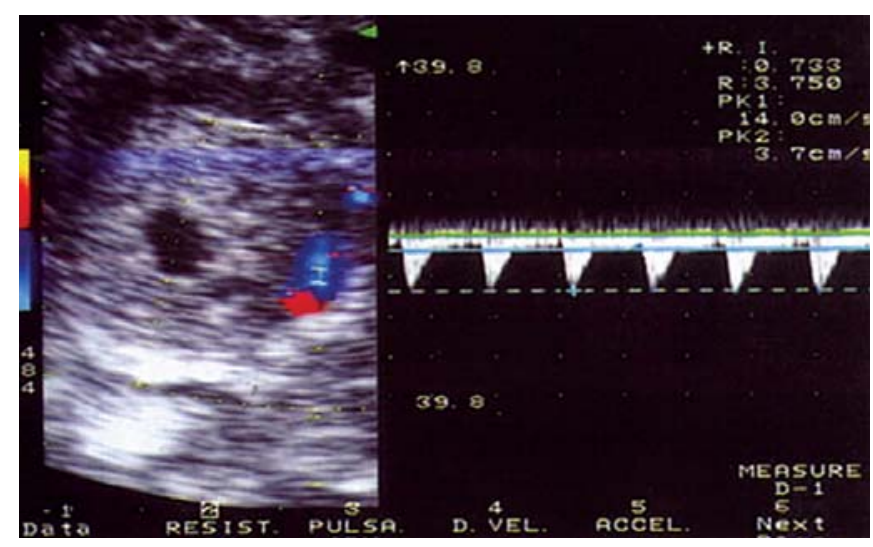

Fig. 5: Gestational sac measuring $12 \mathrm{~mm}$ in the left adnexal region. Color Doppler depicts a small area of angiogenesis characterized by a high resistance index ( $\mathrm{RI}$ of 0.73 ). This finding is typical of tubal abortion

medical management of ectopic pregnancy with methotrexate, and among candidates for medical intervention, to compare and refine the relative efficacy of different regimens ${ }^{9}$ (Figs 4 and 5).

A modern combined hormonal contraceptive containing ethinyl estradiol $20 \mathrm{gm}$ and levonorgestrel 100 gm equates to $1.2 \%$ of the recommended dose of Enovid ${ }^{\circledR}$, the first combined oral hormonal contraceptive marketed in the United States in the mid-60s. It took about two decades in the postmarketing period to establish that such a markedly lower dose was no less effective while providing a greater margin of safety and improved toleration. ${ }^{10}$ Currently, almost all new drug products that are approved for noncyclic use to prevent pregnancy or manage chronic gynecologic conditions are being prescribed for periods longer than they were evaluated in controlled trials. Lybrel being first on the market in May 2007, the primary focus is on venous thrombotic events, but there is still uncertainty about the safety of maintaining the decidua indefinitely. ${ }^{11}$ Ultrasound is widely available to monitor and report effectiveness and complications in women for whom this product is prescribed on an extended basis for any indication, including labeled use for contraception, or off-label use for anovulatory uterine bleeding, polycystic ovary syndrome or endometriosis. It would be of interest to opportunistically correlate ultrasound data with endometrial biopsies and biomarkers.

Despite hopeful preliminary reports, ${ }^{12-15}$ the protective effect of pituitary-ovarian down-regulation with $\mathrm{GnRH}$ analogs such as leuprolide and assumptions that benefits exceed risks remain unproven. ${ }^{16}$ In relation to the agedependent decline in primordial follicle reserves spanning the continual process of atresia, the older the postmenarchal patient the higher the probability of ovarian failure. Given the high-rate of premature ovarian failure in a patient approaching 30 years of age, the potential treatment benefits are likely to be lower, but perhaps more critical if she wishes to conceive. For a patient who is truly intent on conceiving after entering a disease-free period, serious consideration would be given toward a chemotherapeutic regimen that is least deleterious to the ovaries, one that would minimize exposure to cyclophosphamide and platinum-containing agents, for example. Due to possible toxicity of chemotherapy on growing oocytes, a 6-12 month washout period has been suggested prior to attempts to conceive. However, the patient's intention to conceive as early in the disease-free period as possible may be fundamental to the potential success of pituitary-ovarian down-regulation with GnRH. Ultrasound could be used to estimate pretreatment follicle reserve, evidence of suppression and resumption of follicular growth, although biopsy is required to ascertain the ultrastructural integrity of primordial follicles ${ }^{17}$ (Figs 6 to 8 ).

The advancements achieved particularly in the gynecologic and obstetrical application of ultrasound provides unique capacity for physicians who are sensitized and educationally prepared to organize and contribute to the ongoing evaluation of therapies as they are used in actual clinical practice.

\section{REFERENCES}

1. Holbrook A, Keshavjee K, Sebaldt R, Grootendorst P, Levine $\mathrm{M}$, et al. Evaluation of data sources to support pharmacosurveillance. Final Report to Health Policy Research Program, Health Canada. HPRP\#6795-15-2001/4410013, July 2004;30. 


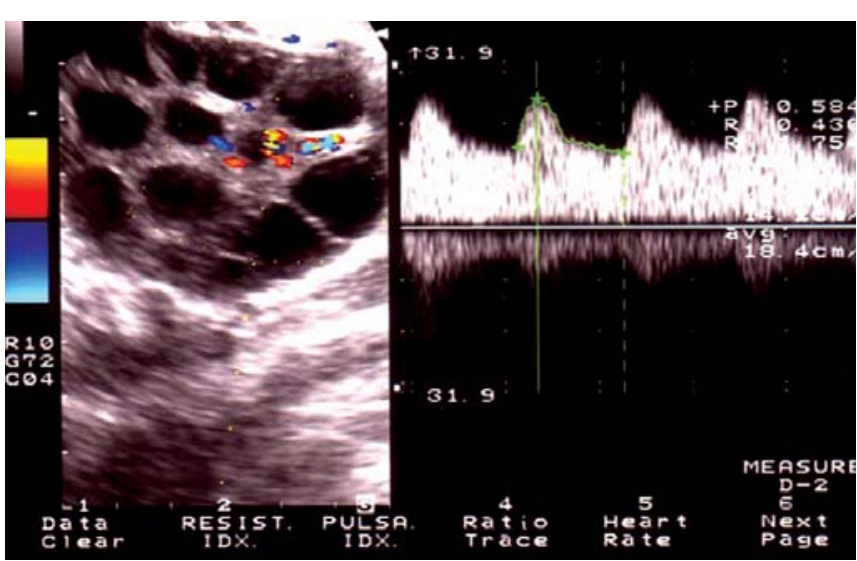

Fig. 6: Early signs of hyperstimulation in a patient with normal ovarian reserve receiving ovulation induction. Color Doppler depicts low vascular impedance blood flow $(\mathrm{RI}$ of 0.43 ) at the periphery of the growing follicles

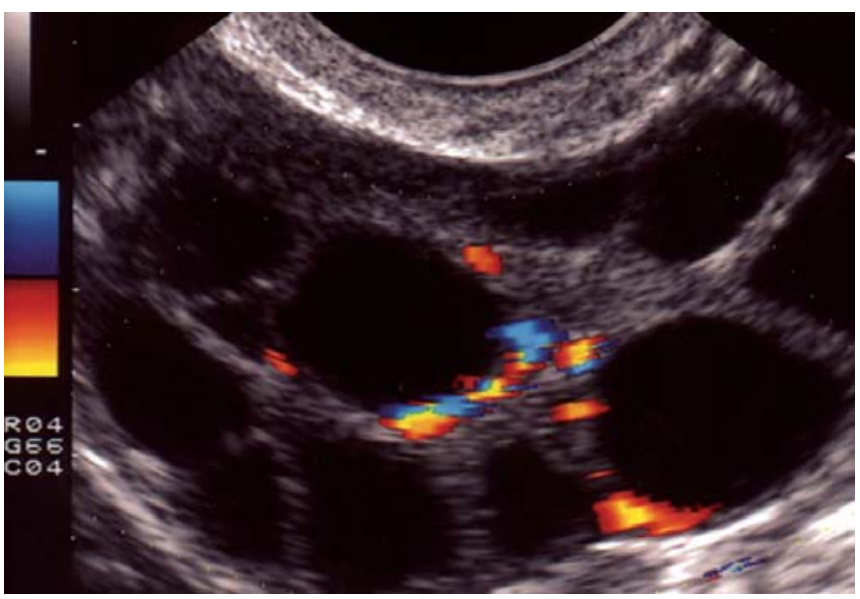

Fig. 7: Color Doppler ultrasound of the ovary undergoing ovulation induction. Ultrasound estimation of the total antral follicle number and intraovarian stomal vascularity achieve high predictive values in prediction of the optimal IVF outcome

2. Carleton B, Foerster V, Waren L, Smith MA. Pharmacosurveillance In Canada: A background paper prepared for the Working Conference on Strengthening the Evaluation of Real World Drug Safety and Effectiveness. August 2005;31.

3. Committee for Medicinal Products for Human Use. Guideline on risk management systems for medicinal products for human use. London: European Medicines Agency, 2005. http:// www.emea.europa.eu/pdfs/human/euleg/9626805en.pdf (accessed 17 September 2009).

4. Ronaldson KJ. The new age of pharmacovigilance. MJA 2009;191(3):132-34.

5. Mann RD. Adverse reactions and the science of pharmacosurveillance. Eur Respir Rev 1998;8(63):1051-53.

6. United States Food and Drug Administration. Prescription Drug User Fee Act (PDUFA) IV. Drug safety five-year plan. Draft. Silver Spring, Md: FDA, 2008. http://www.fda.gov/downloads/ Forindustry/UserFees/PrescriptionDrugUserFee/ ucm093946.pdf (accessed 17 September 2009).

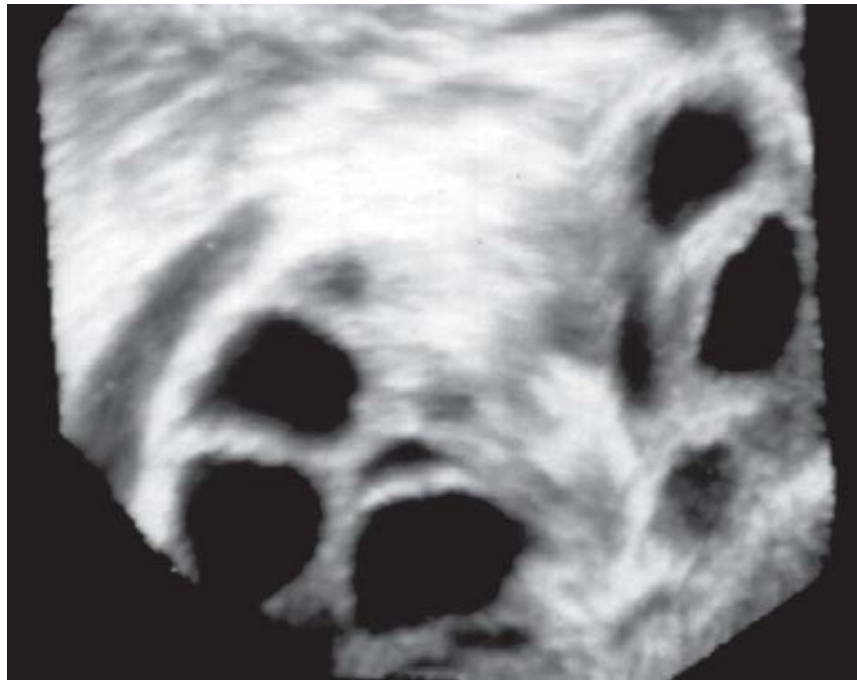

Fig. 8: The same patient. Three-dimensional ultrasound of ovarian hyperstimulation in a patient undergoing ovarian ovulation induction. Multiple developing follicles are clearly visualized

7. Benagiano G, Primiero FM. Uterine leiomyomata: Medical treatment. In: Brosens I (Ed) Uterine fibroids: Pathogenesis and management. London, New York: Taylor and Francis 2006;23753.

8. Kupesic S, Plavsic BM. Ultrasound of uterine fibroids. In: I. Brosens (Ed): Uterine fibroids: Pathogenesis and management. London, New York: Taylor and Francis 2006;139-51.

9. Kupesic S. Ectopic pregnancy. In Donald School Textbook of Ultrasound in Obstetrics and Gynecology 13;230-43.

10. Frazer IS. Forty years of combined oral contraception: The evolution of a revolution MJA 2000;173:541-44.

11. Legro RS, Pauli JG, Kunselman AR, Meadows JW, Kesner JS, et al. Effects of continuous versus cyclical oral contraception: A randomized controlled trial. J Clin Endocrinol Metab 2008;93(2):420-29.

12. Pereya-Pacheco B, Mendez-Ribas JM, Milone G, et al. Use of $\mathrm{GnRH}$ analogs for functional protection of the ovary and preservation of fertility during cancer treatment in adolescents: Preliminary report. Gynecologic Oncology 2001;81:391-97.

13. Recchia F, Sica G, DeFilippis S, et al. Goserelin as ovarian protection in the adjuvant treatment of premenopausal breast cancer: A phase II pilot study. Anticancer Drugs 2002;13:1724.

14. Grundker C, Emons G. Role of gonadotropin-releasing hormone $(\mathrm{GnRH})$ in ovarian cancer. Reprod Biol Endocrinol 2003;65.

15. Blumenfeld Z. Gynecologic concerns for young women exposed to gonadotoxic chemotherapy. Curr Opin Obstet Gynecol 2003;15:359-70.

16. Oktay K, Sönmezer M, Oktem O, Fox K, Emons G, et al. Absence of conclusive evidence for the safety and efficacy of gonadotropin-releasing hormone analogue treatment in protecting against chemotherapy-induced gonadal injury. The Oncologist 2007;12:1055-66.

17. Kupesic S, Kurjak A. Predictors of IVF outcome by threedimensional ultrasound. Hum Reprod 2002;17(4):950-55. 